der Spagat zwischen den Ansprüchen, einerseits das Forschungsfeld der Medienwirkungsforschung möglichst vollständig und systematisch im Überblick darzulegen, anderseits bei den einzelnen Theorien und konkreten Forschungsarbeiten nicht an der Oberfläche zu bleiben, sondern sie im Detail kritisch zu diskutieren. Aus diesem Grund ist das Buch sowohl für den interessierten Sozialwissenschaftler als auch für den im Gebiet forschenden Wissenschaftler von großem Nutzen. Und aus demselben Grund ist es eine sehr gute Empfehlung für alle Studierenden, welche sich mit der empirischen Kommunikationsforschung allgemein oder der Medienwirkungsforschung im Speziellen auseinandersetzen und / oder sich einer Prüfung in diesem Gebiet gegenübersehen.

Und übrigens: Auch wenn ich ebenso wie Michael Schenk einfachen kausalen UrsacheWirkungs-Zusammenhängen skeptisch gegenüberstehe: Es muss wohl doch etwas dran sein, dass ich die erste Auflage dieses Buches vor 15 Jahren zu Beginn meines Studiums geschenkt bekam (Danke, Jens Wolling!) und ich heute als kompetent genug angesehen werde, genau dieses Werk zu rezensieren.

\section{Carsten Wünsch}

\section{Christian Schicha}

\section{Legitimes Theater?}

Inszenierte Politikvermittlung für die Medienöffentlichkeit am Beispiel der „Zuwanderungsdebatte"

Münster: LIT, 2007. - 475 S.

(Studien zur politischen Kommunikation; 1)

ISBN 987-3-8258-0292-9

(Zugl. : Marburg, Univ., Habil.schrift, 2006)

Christian Schicha beschäftigt sich in seiner Habilitationsschrift mit Komponenten der Theatralität innerhalb der massenmedialen Politikvermittlung und der Frage, ob und in welcher Weise die Qualität des politischen Diskurses durch diese beeinflusst wird. Er stellt sein erweitertes Theatralitätskonzept vor, das als geeignetes Analyseinstrumentarium für die Bewertung politischer Mediendiskurse dienen soll. Die Problematik bei der Verwendung von Begriffen wie Theater und Inszenierung im Kontext der Politikvermittlung liegt darin, dass diese bislang sehr vielfältig definiert und operationalisiert worden sind. Eine wichtige Aufgabe in Verbindung mit dieser Thematik ist also, die verwendeten Termini klar zu bestimmen. Daher ist es erfreulich, dass sich der Autor von dem mitunter zugrunde gelegten Alltagsverständnis von Inszenierung und Theatralität distanziert und stattdessen mit einem (theater-)wissenschaftlich begründeten Bedeutungsrahmen operiert.

Die Frage nach der Qualität der Politikvermittlung in den Massenmedien ist elementar und viel diskutiert. Normativen Anforderungen an die Politikberichterstattung stehen journalistische Zwänge in der Praxis (z. B. Verdichtungsdruck) gegenüber, die in der Forschung jedoch oftmals ignoriert werden. Christian Schicha erläutert, warum das demokratietheoretische Ideal in der Praxis nicht erreichbar ist. Er plädiert zu Recht dafür, wissenschaftlich begründete Forderungen der Realität anzupassen, damit Forschungsergebnisse auch für die Praxis relevant werden können.

Der Autor diskutiert zunächst die normativen Grundlagen massenmedialer Politikvermittlung. Es erfolgt eine Auseinandersetzung mit liberalen und deliberativen Öffentlichkeitsmodellen, demokratietheoretisch begründeten normativen Anforderungen sowie der Habermas'schen Diskurstheorie, die laut Verfasser einen Maßstab für die Angemessenheit von Inhalten liefern kann. Obwohl das Phänomen Öffentlichkeit schwer operationalisierbar sei und die diskutierten Modelle die Strukturen, Prozesse und Eigenlogiken sowohl der Medien als auch der Politik nicht berücksichtigen würden, könnten diese als Heuristik für eine empirische Analyse öffentlicher Kommunikationszusammenhänge dienen. So sollten die Idealnormen weiterhin als Maßstab für die Angemessenheit der Politikvermittlung gelten, Sachzwänge in der Praxis müssten jedoch bei der Beurteilung berücksichtigt werden. Insgesamt bietet diese Abhandlung einen gut strukturierten, verständlichen Überblick über das bisweilen recht unübersichtliche Themenfeld Öffentlichkeit.

Weiterhin gibt der Autor eine gute Übersicht über die relevanten Modelle zur Beschreibung des Verhältnisses von Medien und Politik sowie die wechselseitigen Abhängigkeiten der beiden Systeme. Die Ebenen des Politikvollzugs, der Politikvermittlung und deren Zusammenhang mit Inszenierung wird thematisiert. Auf den Stufen der Herstellungs- und Darstellungs- 
politik differenziert der Autor Inszenierung bzw. Theatralität: Demzufolge ist die Inszenierungsintensität auf der Ebene der medialen Politikdarstellung am stärksten. Auch der Grad der Theatralisierung steige sukzessive, da zugleich der Öffentlichkeitsbezug ansteige und die strukturellen Medienzwänge dazu führten, dass Aufmerksamkeitserzeugung auch durch theatralische bzw. unterhaltende Elemente forciert würde. Neben Dramatisierung und Emotionalisierung sowie der Erfüllung von Nachrichtenfaktoren mittels der Inszenierung von Pseudo-Ereignissen sei Unterhaltung ein wesentlicher Inszenierungsmechanismus zur Aufmerksamkeitserzeugung. Diesem Aspekt widmet sich der Autor sehr ausgiebig; auch der Exkurs zur Kritischen Medientheorie und ihren Nachfolgekonzepten - kompakt, übersichtlich und leicht verständlich dargestellt - zielt auf die Beurteilung populärer Medieninhalte und die Debatte über Chancen und Gefahren unterhaltungsorientierter Angebote. Nach Schichas Verständnis erfüllt Unterhaltung eine wichtige Funktion im Politikvermittlungsprozess, sie kann sogar zentrale Bedingung für einen erfolgreichen Verlauf sein. Auch unterhaltsame Formen der Politikvermittlung könnten durchaus angemessen sein. Dabei dürfe allerdings die „Form“ (Unterhaltung) nicht den Inhalt ersetzen (S. 369).

Ein weiteres Kapitel widmet sich dem erweiterten Theatralitätskonzept, das als Grundlage für Analysen von Politikvermittlung dienen soll. Im Zentrum steht die These der Theatralisierung der politischen Kommunikation. Die von den Medien und den politischen Akteuren angewendeten Strategien könnten als Dramatisierungsstrategien angesehen werden, die wiederum dem Phänomen der Theatralität zuzuordnen seien. Theatralität wird hier als sozialwissenschaftliche Analysekategorie verstanden, da sie sich auf soziale Interaktionen bezieht, die sich an den Kategorien theatralischen Handelns ausrichten. Schicha überträgt die Theatralitätskomponenten Inszenierung, Korporalität, Performance und Wahrnehmung mit Blick auf die massenmediale Politikvermittlung. Das ursprünglich aus der Theaterwissenschaft stammende Konzept ist aus Vorarbeiten des Autors bereits bekannt. In Bezug auf die eingangs erwähnte wichtige begriffliche Abgrenzung unterscheidet Schicha die journalistische Inszenierung vom mit Fiktionalität verbundenen Begriff des Theaters und dem alltagssprachlichen Begriffsgebrauch. Inszenierung ist seiner Ansicht nach aufgrund der Komplexität moderner Gesellschaften und der Selektionslogik / -optik der Medien unumgänglich und legitim. Inszenierung sei zunächst eine wertfreie analytische Kategorie zur Beschreibung des Einsatzes von Techniken, um ein Ereignis für ein Publikum zur Erscheinung zu bringen. Diese Techniken wiederum könnten sowohl aufklärerisch als auch manipulativ angelegt sein. Unklar bleibt leider an dieser Stelle, wie solche jeweils möglichen Richtungen empirisch messbar gemacht werden können. Gleiches gilt für die übrigen Theatralitätskomponenten sowie die Ergänzungen Ritualisierung und Symbolisierung: Rituale und Symbole seien Schicha zufolge dann Schein oder Täuschung, wenn keine Argumentation mehr erfolge, sondern es nur noch um positive Eindrücke beim Rezipienten gehe. Auch hier bleibt unklar, wie dies zu bemessen ist.

Der Autor schlussfolgert aus seinen theoretischen Überlegungen, dass die Qualität theatraler (Medien-)diskurse nur anhand von Einzelfallprüfungen beurteilt werden kann. In einer qualitativen Inhaltsanalyse untersucht er exemplarisch die Berichterstattung zur sog. „Zuwanderungsdebatte “ in verschiedenen TV- und Printmedien. Im Mittelpunkt steht der inszenierte Eklat bei der Abstimmung im Bundesrat Anfang 2002. Auf der Ebene der Darstellungspolitik und der medialen Politikdarstellung wird der politische, informative, theatralische und unterhaltende Gehalt der Politikvermittlung in den politischen Mediendiskursen ermittelt. Interessant ist die Tatsache, dass der Ort für das „legitime Theater“, das Parlament, bisher eher Hinterbühne als Vorderbühne war und in diesem Fall massiv für die Inszenierung genutzt wurde. Hier vermischten sich die Ebenen der Politikherstellung und -darstellung; Schicha selbst spricht von einer Verzerrung der parlamentarischen Kommunikation. Als Ergebnis der Analyse bezeichnet er das Diskursniveau auf der Ebene der Darstellungspolitik als ambivalent: Er konstatiert einen hohen Grad der inszenierten Emotionalisierung; ein Spannungsbogen wurde aufgebaut, Nachrichtenfaktoren bedient, Polarisierung und Diffamierung betrieben und metaphorische Sprache verwendet. Zugleich wurden aber auch die zentralen Argumente ausgetauscht und die unterschiedlichen Positionen herausgestellt. Im Groben lässt sich dies auf die Analyse der 
Berichterstattung übertragen, wenngleich sich große Unterschiede zwischen den untersuchten Medien zeigen. So zeichnen sich die Boulevardzeitungen durch einen hohen Unterhaltungs- und Theatralitätsgehalt, aber einen niedrigen Informations- und Politikgehalt aus. Bei den untersuchten Qualitätszeitungen verhält es sich umgekehrt. In den Nachrichtenformaten ist das Verhältnis in etwa ausgeglichen. In Talkshows dominiert der Unterhaltungsaspekt, wobei das Diskursniveau stark von der Art der Moderation abhängt.

Die Untersuchung ist leider sehr grob konzipiert - dadurch wird nicht widergespiegelt, dass das erweiterte Theatralitätskonzept ein vielversprechendes Analyseinstrument zur Beurteilung der Qualität von Politikvermittlung ist. Wie der Autor selbst in seinem Fazit anmerkt, scheint es dringend nötig, die hier verwendeten vier Kategorien auszudifferenzieren und die Betrachtung über verbale Elemente hinaus auf solche nonverbaler bzw. visueller Art auszuweiten.

Das Buch bietet über das vielversprechende Theatralitätskonzept hinaus einen hervorragenden Einstieg in sowohl das Themenfeld der massenmedialen Politikvermittlung als auch die noch recht unstrukturierte Forschungsperspektive der Inszenierung und Theatralität. Einen Beitrag dazu leisten insbesondere die gut strukturierten und kompakten Überblicke zu den einzelnen theoretischen Komplexen, die mit dem Inszenierungs- bzw. Theatralitätsblickwinkel auf Medieninhalte in Verbindung gebracht werden können.

Andrea Burmester

\section{Thomas Schierl (Hrsg.)}

\section{Prominenz in den Medien}

Zur Genese und Verwertung von Prominenten in Sport, Wirtschaft und Kultur

Köln: Halem, 2007. - 359 S.

ISBN 978-3-938258-61-3

"In the future, everyone will be world-famous for 15 minutes" (Andy Warhol, 1968). Angesichts des heutigen Stellenwerts von Prominenz erscheint die damalige Prognose von Andy Warhol durchaus realistisch: Ganze Zeitschriften (z. B. „Gala“) und Fernsehsendungen (z. B. „Prominent“) widmen sich dem Thema Prominenz, TV-Formate mit dem Prä- fix „Promi-“ im Titel präsentieren mehr oder weniger bekannte Persönlichkeiten beim Kochen, Essen, Heiraten, Tanzen, Eiskunstlaufen, Drogenentzug oder Operiert-Werden, immer mehr Prominente tauchen in der Werbung auf und andauernd werden neue Promis und Stars gesucht. Zumindest der fleißige Medienkonsument kann den Eindruck gewinnen, als gäbe es inzwischen mehr Prominente als "normale“ Menschen. Trotz seiner hohen Publizität ist das Thema Prominenz jedoch bislang - sieht man einmal von der Arbeit von Birgit Peters aus den Jahr $1996 \mathrm{ab}$ - zumindest im deutschsprachigen Raum kaum Gegenstand wissenschaftlichen Interesses gewesen. Der von Thomas Schierl herausgegebene Reader erscheint daher zur richtigen Zeit, um den großen weißen Fleck auf der Forschungslandkarte etwas kleiner werden zu lassen.

Der Band ist das Ergebnis eines Workshops („Stars, Helden und Möchtegerne“) des Instituts für Sportpublizistik der Deutschen Sporthochschule Köln, von dem der Herausgeber sich wünscht, dass er die Befunde zum Themenkomplex Prominenz einem größeren, interessierten Publikum zugänglich und das Phänomen im wissenschaftlichen Diskurs prominenter machen kann (S. 8). Das Potenzial des Readers, zu dem insgesamt 22 Autoren aus unterschiedlichen Disziplinen 14 Aufsätze beitrugen, ist durchaus geeignet, um diese beiden Wünsche in Erfüllung gehen zu lassen.

In den durchweg verständlich und angenehm lesbar geschriebenen Beiträgen wird das Phänomen „Prominenz" aus unterschiedlichen theoretischen Perspektiven analysiert. Unter der Überschrift „Prominenz als Medieninhalt" zeigt Thomas Schierl anhand inhaltsanalytischer Ergebnisse, welche Rolle Prominente als „ökonomisch wichtiger Rohstoff“ (S. 7) für die Medien spielen und wie die Berichterstattung an die Bedürfnisse der Rezipienten angepasst wird. Bosshart und Witmer beschreiben, wie die mediale Präsentation der Miss Schweiz für alle Beteiligten zu einer Win-Win-Situation führen kann. Im Rahmen eines medienkulturtheoretisch orientierten Beitrags nimmt Christoph Jacke eine Typologisierung von Prominenten in Stars, Anti-Stars und Anti-Star-Stars vor und schließlich informiert Jan Räker in seinem Beitrag unter einer juristischen Perspektive über die Konsequenzen der sog. „Caroline-Entscheidung " des EGMR für die deutsche Rechtsprechung über die Veröffentlichung von 